\title{
Exploring the prognostic value of SCARF1 in non-small cell lung cancers
}

\author{
Daniel A. Patten ${ }^{1 *}$ and Shishir Shetty ${ }^{1}$ \\ ${ }^{1}$ National Institute for Health Research Birmingham Liver Biomedical Research Unit \\ and Centre for Liver and Gastrointestinal Research, Institute of Immunology and \\ Immunotherapy, University of Birmingham, Birmingham, United Kingdom
}

Running title: SCARF1 in lung cancers

\section{"Corresponding author:}

Dr Daniel Patten

National Institute for Health Research Birmingham Liver Biomedical Research Unit and Centre for Liver Research

Institute of Immunology and Immunotherapy

University of Birmingham

Birmingham

B15 2TT

United Kingdom

Email: d.a.patten@bham.ac.uk

Tel: +441214158692

Fax: +441214158701

Keywords: scavenger receptor, NSCLC, lung adenocarcinoma, tumour microenvironment, lymphocyte recruitment 


\section{Abstract}

Scavenger receptor class $\mathrm{F}$ member 1 (SCARF1) has previously been shown to be highly expressed within the human liver, hold prognostic value in hepatocellular carcinoma and mediate the specific recruitment of leukocytes to liver sinusoidal endothelial cells; however, to date, the liver remains the only major organ in which SCARF1 has been explored in any detail. Here, we utilised publically-available RNAsequencing data from The Cancer Genome Atlas (TGCA) to identify the lungs as a site of significant SCARF1 expression and attribute the majority of its expression to endothelial cell populations. Next, we show that SCARF1 expression is significantly reduced in two histologically distinct types of non-small cell lung carcinoma cancers (NSCLCs), lung adenocarcinoma (LUAD) and lung squamous cell carcinoma (LUSC), compared to non-tumoural tissues. Interestingly, loss of SCARF1 expression was associated with aggressive tumour biology in LUAD tissues, but not in LUSC. Furthermore, increased SCARF1 expression was highly prognostic of better overall survival in LUAD tumour tissues, but this was again in contrast to LUSC tumours, in which SCARF1 held no prognostic value. Finally, we showed that SCARF1 is widely expressed in tumour endothelial cells of non-small cell lung cancers and that its total expression in LUAD tumour tissues correlated with immune score and $\mathrm{CD}^{+} \mathrm{T}$ cell infiltration. This study represents the first detailed exploration of SCARF1 expression in normal and diseased human lung tissues and further highlights the prognostic value and therapeutic potential of SCARF1 in immunologically active cancers. 


\section{Introduction}

Globally, lung cancer is the leading cause of cancer mortality and is responsible for more than 1.7 million deaths worldwide each year [1]. This is largely due to the fact that the 5-year survival from lung cancer ranges from 4-17\%, depending on cancer stage and regional variation [2]. Non-small cell lung cancers (NSCLCs) make up the majority ( $85 \%$ ) of lung cancers [3]; of those, lung adenocarcinoma (LUAD) is the most common histologic subtype, accounting for about $40 \%$ of total lung cancer incidence [4]. The second most prevalent subtype of NSCLC is lung squamous cell carcinoma (LUSC), which accounts for around $30 \%$ of the global lung cancer incidence [4]. The high mortality rate in lung cancers is largely due to the fact that many patients present with advanced disease at diagnosis, which often includes the presence of metastatic disease [3]. In early stage disease, surgical resection remains the single most effective treatment [2]; in cases where surgery is not a viable option, for example in later stage diseases, combination therapies of thoracic radiotherapy and non-specific chemotherapies have traditionally been used [2]. However, lung cancers are notoriously heterogeneous diseases [5,6] and understanding their molecular make-up is crucial for the development of more targeted therapies. Over the last $20+$ years, genetic testing has resulted in a number of highly specific therapies being developed and survival time of patients have improved [2]; nevertheless, furthering our understanding of the tumour immune microenvironment is still pertinent to the development of novel biomarkers and therapies in NSCLCs. Much of the research to date has predominantly focused on immune cell profiles $[7,8]$ and stromal (e.g. fibroblast) populations $[9,10]$ of NSCLC tumours, whilst other tumour-resident cells, such as endothelial cells, are less well studied. We have previously described the prognostic value of the endothelial- 
expressed scavenger receptor class F, member 1 (SCARF1) in hepatocellular carcinoma (HCC) and hypothesised that it plays a key role in the recruitment of proinflammatory $\mathrm{CD}^{+} \mathrm{T}$ cells to the HCC tumour microenvironment [11]; here, we sought to study SCARF1 in the context of NSCLCs.

Scavenger receptors are a large super-family of proteins which are defined by their ability to bind and internalise a vast range of endogenous and exogenous ligands and removing them from the general circulatory system [12]. Consequently, scavenger receptors represent a major subset of innate pattern recognition receptors (PRRs) and are known to key roles in tissue homeostasis, infections and inflammatory diseases [12]. Given the high environmental antigenic load that is inhaled with each breath, it is unsurprising that a number of scavenger receptors are expressed in resident cell populations of the lung. In particular, the Class A family of scavenger receptors is known to play a key role in innate immunity in response to a number of microbial infections [13,14], environmental nanoparticles and allergens $[15,16]$ and inhaled oxidants [17]. SCARF1 has also previously been confirmed to be expressed in human [18] and murine [19,20] lung tissues. SCARF1 was first identified in cDNA libraries from human umbilical vein endothelial cells (HUVEC) [21] and exhibits high expression in primary human liver sinusoidal endothelial cells (LSEC) [22], which are also known to be exposed to high levels of antigenic matter from the gut. SCARF1 has been shown to bind and internalise a wide range of endogenous and exogenous ligands [23], such as apoptotic host cells [24] and viral [25-27], fungal [19] and bacterial [28-31] antigens. Nevertheless, its expression in human lung tissues and in cancers of the lung have not been explored in any detail to date. 
Here, through the utilisation of the publically-available TGCA (The Cancer Genome Atlas) datasets (http://cancergenome.nih.gov), we initially described the expression of SCARF1 in a range of human tissues and demonstrate lung tissues as a major site of expression. Next, we showed that the expression of SCARF1 within lung tissues is largely associated with endothelial cell populations. Subsequently, we described a downregulation of SCARF1 expression in two distinct histological types of non-small cell lung carcinoma tumours, lung adenocarcinoma (LUAD) and lung squamous cell carcinoma (LUSC), compared to non-tumourous control tissues. Following this, we explored the relationship of SCARF1 expression with tumour progression and, consequently, found an association with loss of SCARF1 expression with aggressive tumour biology in LUAD tumours, but not in LUSC tumour tissues. Following this, we evaluated the prognostic value of SCARF1 expression in LUAD and LUSC tumours by generating survival curve data, via KM Plotter (http://kmplot.com/analysis/). In support of the pathological findings, high SCARF1 expression in LUAD tumour tissues was found to correlate with a better overall survival; however, SCARF1 expression showed no prognostic value in LUSC tumours. In addition, through use of the LungECtax database (https://endotheliomics.shinyapps.io/lung ectax/), we demonstrated SCARF1 expression across a range of of endothelial cell populations present in both normal lung and NSCLC tumour tissues. Finally, we used publically-available tools, Estimation of STromal and Immune cells in MAlignant Tumor tissues using Expression data (ESTIMATE; https://bioinformatics.mdanderson.org/estimate/) and Tumor IMmune Estimation Resource (TIMER; https://cistrome.shinyapps.io/timer/) to correlate SCARF1 expression with immune score and the level of $\mathrm{CD}^{+}{ }^{+}$cell infiltration, respectively, in LUAD tumour tissues. Using ESTIMATE, we 
demonstrated a moderate positive correlation between SCARF1 expression and immune infiltration score and, via TIMER, we confirmed that SCARF1 expression correlates more specifically with $\mathrm{CD}^{+} \mathrm{T}$ cell infiltration. Our results demonstrate that SCARF1 could be a prognostic biomarker in LUAD and that lung-expressed SCARF1 could potentially function to alter the inflammatory status of the tumour microenvironment. 


\section{Materials and Methods}

In silico data analysis

Publically-available RNA-sequencing data was utilised throughout this study and several publically-available tools were used in its analysis. To explore SCARF1 expression in range of human major organs and in lung tumour and relevant nontumourous tissue controls, data was obtained from the Genotype-Tissue Expression (GTEx) and The Cancer Genome Atlas (TGCA) datasets, via the University of California Santa Cruz (UCSC) Xena tool (https://xenabrowser.net/). SCARF1 expression data in various cell populations of the human lung was generated by the LungMAP Consortium and downloaded from (www.lungmap.net) (accessed on 4th June 2020). Correlation of SCARF1 expression with tumour progression/aggression was performed via the cBioPortal website (https://www.cbioportal.org/) (accessed $28^{\text {th }}$ April 2020). With the use of the publically-accessible tool KM Plotter (http://kmplot.com/analysis/), survival data was generated using the Affymetrix ID 206995_x_at (SCARF1). Data was censored at a 60-month threshold and was split into two groups ('High' and 'Low') by the median of SCARF1 expression. Resultant data was exported to Prism ${ }^{\circledR} 6$ software (GraphPad Software Inc.) and survival curves were produced. tSNE plots of SCARF1 expression in normal endothelial cells (NEC) and tumour endothelial cells (TEC) were generated by the publicallyavailable LungECtax database (https://endotheliomics.shinyapps.io/lung ectax/; accessed on $4^{\text {th }}$ June 2020). Immune infiltration scores of LUAD tumours were correlated with SCARF1 expression via Estimation of STromal and Immune cells in MAlignant Tumor tissues using Expression data (ESTIMATE; https://bioinformatics.mdanderson.org/estimate/; accessed $19^{\text {th }}$ August 2020). Level of $\mathrm{CD}^{+} \mathrm{T}$ cell infiltration of LUAD tumours was correlated with SCARF1 expression 
via the Tumor IMmune Estimation Resource (TIMER;

https://cistrome.shinyapps.io/timer/; accessed 12th May 2020).

\section{Statistical analyses}

All data were tested for normal distribution by the D'Agostino-Pearson omnibus test.

All data were found to be non-parametric and so were expressed as median \pm interquartile range $(\mathrm{IQR})$, with the number of experimental repeats $(n)$ specified in each case. For single comparisons, statistical significance was determined by MannWhitney $U$-test, whereas evaluation of multiple treatments was performed by Kruskall-Wallis one-way analysis of variance with post hoc Dunn's test. Matched data was analysed by a Wilcoxon signed-rank test. A $p$-value of $\leq 0.05$ was considered as statistically significant. All statistical analyses were undertaken using Prism ${ }^{\circledR} 6$ software (GraphPad Software Inc.). 


\section{Results}

SCARF1 is highly expressed in human lung tissues

We have previously shown that SCARF1 is highly present in in human liver tissues $[11,22]$; however, the exploration of its expression in other major organs is limited. Here, through the analysis of the publically-available TCGA RNA sequencing data and using the expression in the liver as a reference, we showed that SCARF1 was differentially expressed at the gene level in a wide range of human major organs. In the 14 major organs explored, 8 (pancreas, brain, skin, bladder, kidney, esophagus, stomach and colon) demonstrated significantly lower expression than the liver (Figure 1A), whereas 4 (heart, breast, lung and spleen) exhibited significantly higher expression (Figure 1A); the only exception was the small intestine, which showed comparable SCARF1 expression to the liver. Of the tissues explored, the lungs exhibited marked SCARF1 expression, with only the levels in the spleen found to be higher. Through the use of additional publically-available RNA sequencing data generated from bulk cell populations, we show that endothelial cell populations account for the vast majority of SCARF1 expression in the human lung at all stages of the life cycle (neonate-infant-child-adult), when compared with epithelial, mesenchymal and immune cell populations (Figure 1B).

SCARF1 expression is downregulated in NSCLC

We have previously shown that SCARF1 is downregulated in gastrointestinal cancers, such as hepatocellular carcinoma [11], and, given the high expression in the human lung, we next explored its expression in non-small cell lung carcinomas (NSCLCs). Analysis of the publically-available RNA-sequencing data from The 
Cancer Genome Atlas (TGCA) and Genotype-Tissue Expression (GTEx) project showed that SCARF1 expression is significantly $(p \square \leq \square 0.001)$ lower in both LUAD and LUSC tumour tissues in comparison to normal lung tissues (Figure 1C). The downregulation of SCARF1 expression was also evident when we compared tumour tissues with matched non-tumourous tissues from the same donor in both LUAD (Figure 1D) and LUSC (Figure 1E). 
A

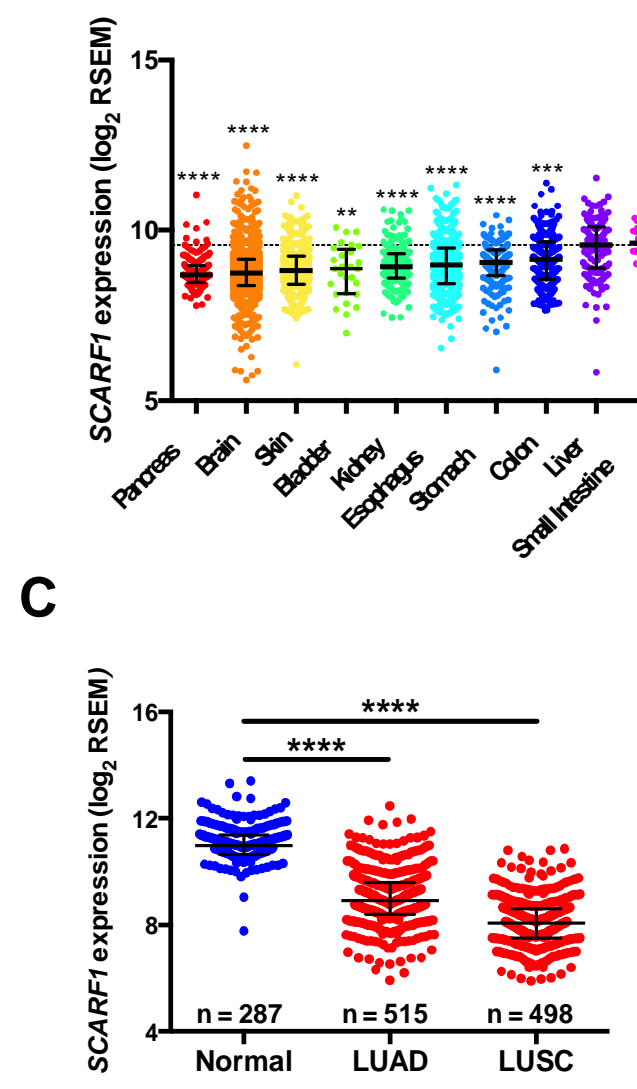

B

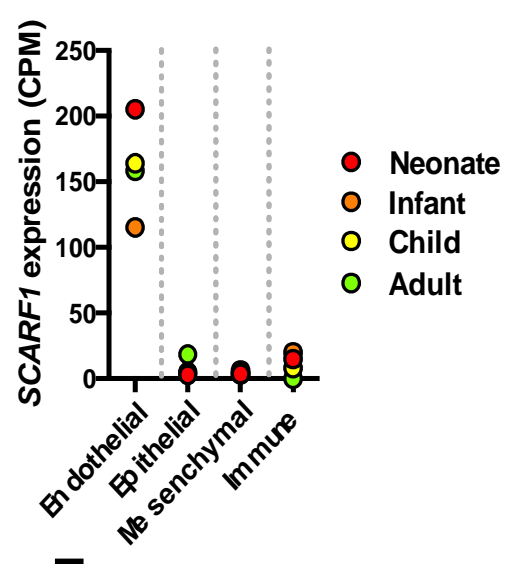

D

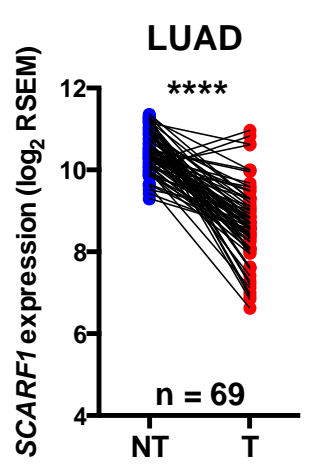

E

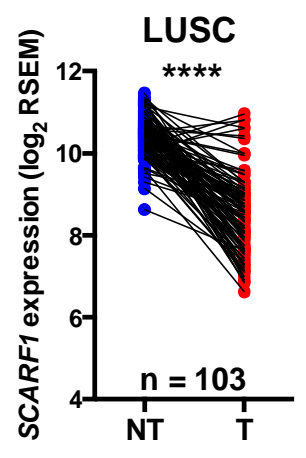

Figure 1 - SCARF1 mRNA expression is high in lung tissues, but downregulated in lung cancers. (A) Expression of SCARF1 gene expression in major tissues: pancreas $(n=169)$; brain $(n=1136)$; skin $(n=556)$; bladder $(n=28)$; kidney $(n=167)$; esophagus $(n=662)$; stomach $(n=208)$; colon $(n=345)$; liver $(n=$ 160); small intestine $(n=92)$; heart $(n=376)$; breast $(n=292)$; lung $(n=396)$; spleen $(\mathrm{n}=99) .{ }^{*},{ }^{* *},{ }^{* * *}$ and ${ }^{* * * *}$ are representative of statistical significance as measured by the Kruskal-Wallis test, where $p \square \leq \square 0.05, p \square \leq \square 0.01, p \square \leq \square 0.005$ and $p \square \leq \square 0.001$, respectively. RSEM = RNA-Seq by Expectation Maximization. The dotted line is representative of the SCARF1 expression in Liver. (B) SCARF1 gene expression in different bulk cell populations of neonatal (red), infant (orange), child (yellow) and adult (green) human lung. CPM = counts per million. (C) Comparison of SCARF1 gene expression in normal lung tissues with lung adenocarcinoma (LUAD) and lung squamous cell carcinoma (LUSC) tumour tissues. ${ }^{* * * *}$ indicates statistical significance as measured by the Kruskal-Wallis test, where $p \square \leq \square 0.001$. (D and $E$ ) Comparison of SCARF1 gene expression in non-tumoural (NT) tissues with matched tumoural (T) tissues in both LUAD and LUSC. ${ }^{* * *}$ indicates statistical significance as measured by a Wilcoxon signed-rank test, where $p \square \leq \square 0.001$. Data in (A) and (C-E) was generated from the TGCA dataset using the University of California Santa Cruz (UCSC) Xena tool (https://xenabrowser.net/). Data in (B) are based upon data generated by the LungMAP Consortium and downloaded from (www.lungmap.net), on $4^{\text {th }}$ June 2020 . The LungMAP consortium and the LungMAP Data Coordinating 
bioRxiv preprint doi: https://doi.org/10.1101/2021.01.28.428617; this version posted January 28, 2021. The copyright holder for this preprint (which was not certified by peer review) is the author/funder, who has granted bioRxiv a license to display the preprint in perpetuity. It is made available under aCC-BY-NC 4.0 International license.

Center (1U01HL122638) are funded by the National Heart, Lung, and Blood Institute (NHLBI). 
Loss of SCARF1 expression is associated with more advanced and aggressive tumours in LUAD, but not LUSC

Previously, we have shown that a loss of SCARF1 expression in hepatocellular carcinoma tumours is associated with more aggressive tumour biology [11]; here, we aimed to utilise the TGCA dataset to explore whether this was also the case in NSCLCs. Firstly, we explored SCARF1 expression levels in cases of NSCLCs at different stages of the disease, from early stage disease (Stage I) through to highly developed and metastatic disease (Stage IV). When compared to patients with Stage I disease, cohorts of LUAD patients with Stages II, III and IV disease all demonstrated a trend for decreased SCARF1 expression; however, only the data for the Stage II cohort was calculated to be statistically significant ( $p \square \leq \square 0.005$ ). We next correlated SCARF1 expression with other parameters commonly associated with tumour aggressiveness; in particular, we focussed on Aneuploidy Score [33] and Buffa Hypoxia Score [34]. We demonstrated a moderate negative correlation of SCARF1 expression with both Aneuploidy Score (Figure 2B) and Buffa Hypoxia Score (Figure 2C) in LUAD tumour tissues, thus providing further evidence that a loss of SCARF1 expression is associated with more adverse tumour biology. In stark contrast, patient cohorts with LUSC all showed comparable SCARF1 expression levels, regardless of disease stage (Figure 2D). In addition, the negative correlations between SCARF1 expression levels and Aneuploidy Score and Buffa Hypoxia Score were much weaker in LUSC tumour tissues (Figure 2E and 2F), when compared to those seen in LUAD tumours (Figures 2B and 2C). 
A

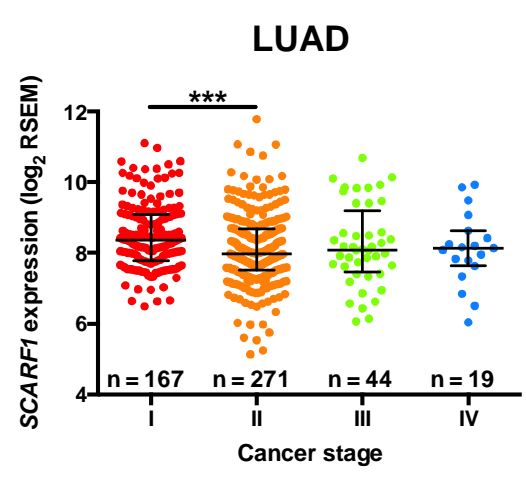

B

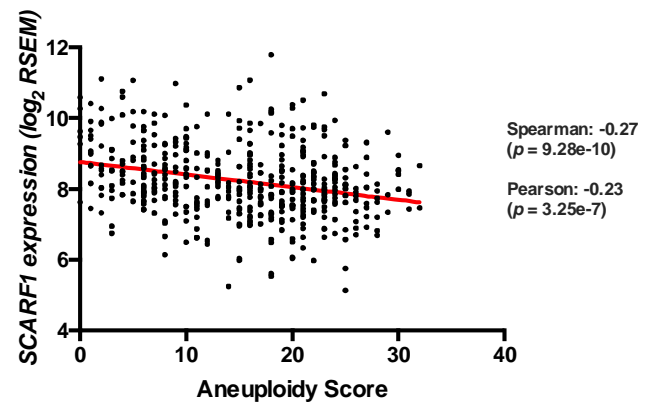

C

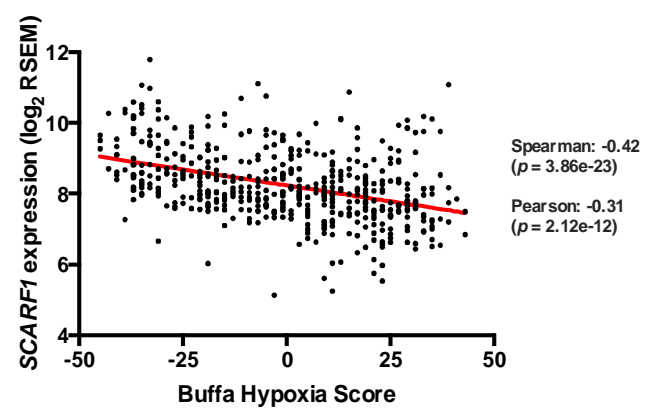

D

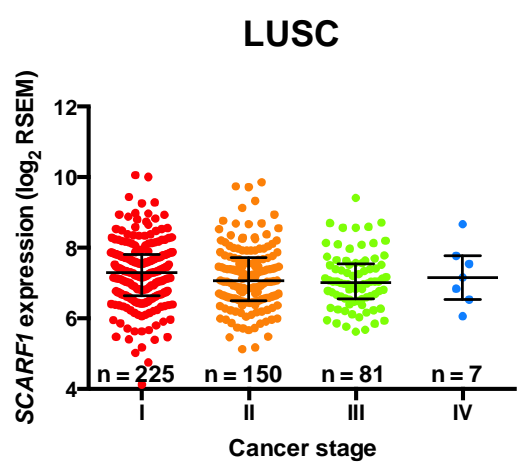

E

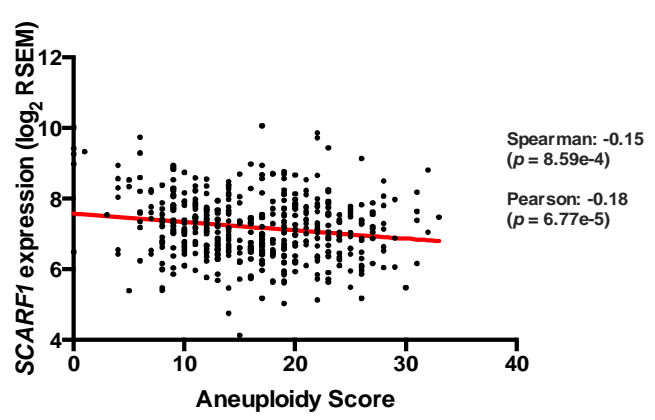

$\mathbf{F}$

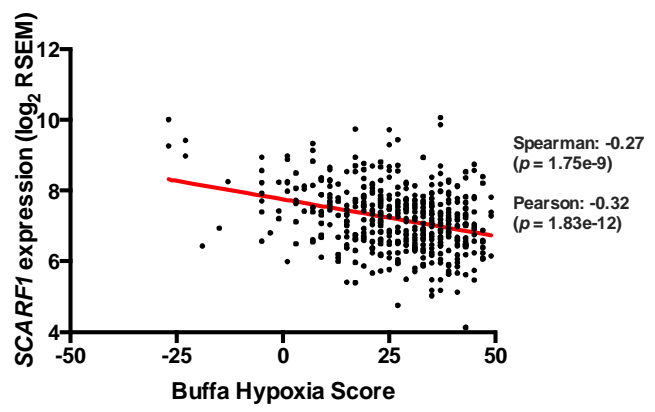

Figure 2 - More advanced and aggressive LUAD tumours exhibit lower SCARF1 expression. (A) SCARF1 expression in LUAD tumour tissues from the four cancer stages. ${ }^{* * *}$ is representative of statistical significance as measured by the Mann Whitney $U$-test, where $\mathrm{p} \square \leq \square 0.005$. SCARF1 expression in LUAD tumours correlated to tumour aggression parameters $(B)$ Aneuploidy score $(n=493)$ and $(C)$ Buffa Hypoxia score $(\mathrm{n}=503)$. (D) SCARF1 expression in LUSC tumour tissues from the four cancer stages. SCARF1 expression in LUSC tumours correlated to tumour aggression parameters (E) Aneuploidy score $(n=465)$ and $(F)$ Buffa Hypoxia score $(n=466)$. Data in this Figure was generated from the TGCA dataset using the cBioPortal website (https://www.cbioportal.org/) (accessed 28 ${ }^{\text {th }}$ April 2020). 
Loss of SCARF1 expression is associated with worse outcomes in LUAD, but not LUSC

We next aimed to explore if SCARF1 expression was in any way associated with outcome in either histological subtype of NSCLC. Firstly, we explored disease-free status since initial treatment in LUAD and showed that patients whose disease recurred or progressed had significantly ( $p \square \leq \square 0.05)$ lower expression levels of intratumoutal SCARF1 expression than those who remained disease-free (Figure 3A). Following this, we explored SCARF1 expression and disease-specific survival status in LUAD patients; SCARF1 expression in tissues from those patients who subsequently died with a tumour burden was significantly ( $p \square \leq \square 0.005$ ) lower than patients who remained alive or died tumour-free (Figure 3B). We next explored the same parameters in LUSC patients. We demonstrated comparable levels of SCARF1 between patients whose disease recurred/progressed and those who remained disease-free (Figure $3 C$ ) and between those patients who subsequently died with a tumour burden and those who remained alive or died tumour-free (Figure 3D). 


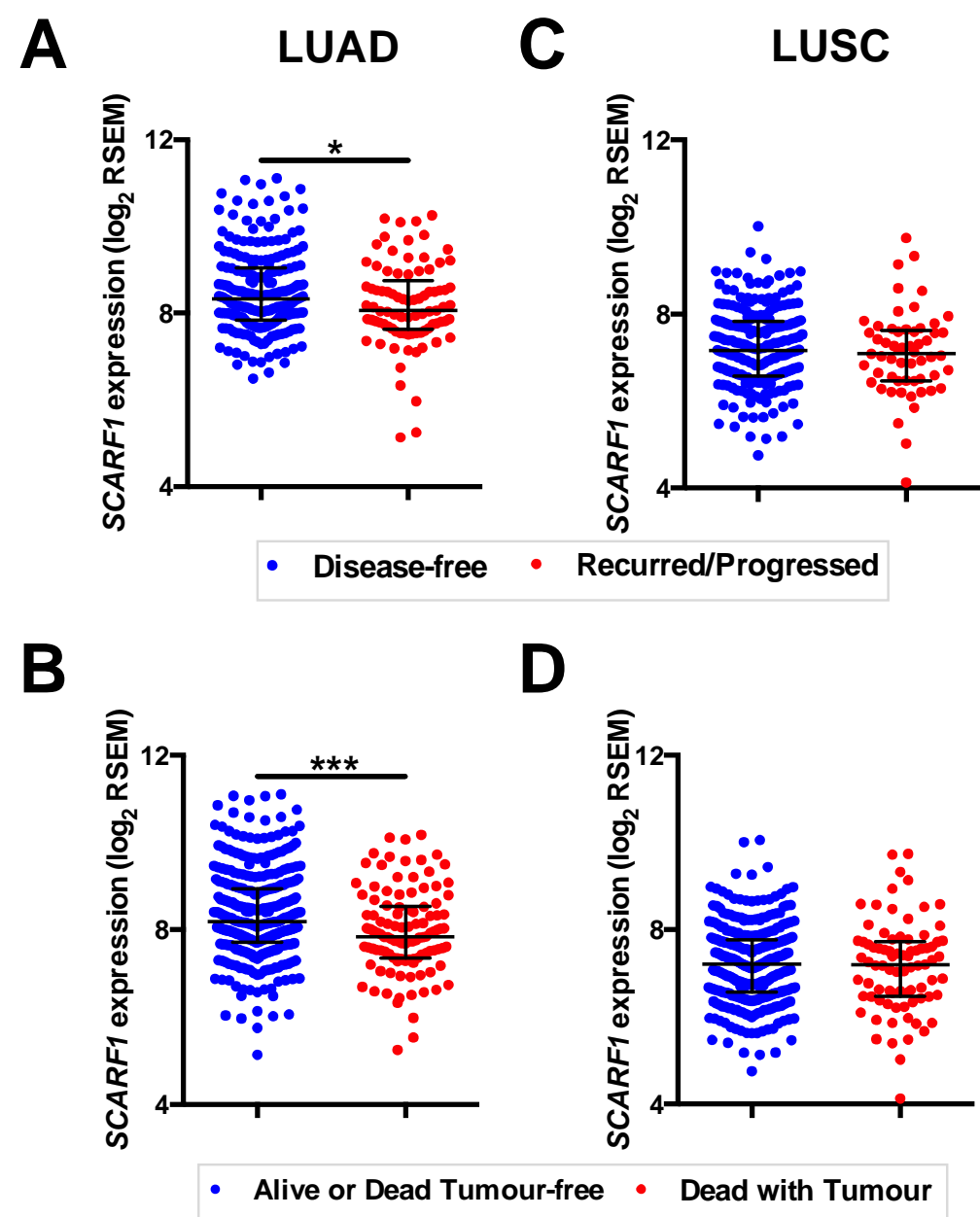

Figure 3 - Lower SCARF1 expression is associated with worse outcomes in LUAD, but not LUSC. Comparison of SCARF1 expression in (A) LUAD and (C) LUSC patients who survived and remained disease-free (blue dots) and those in which disease recurred or progressed (red dots). ${ }^{*}$ is representative of statistical significance as measured by the Mann Whitney U-test, where $\mathrm{p} \square \leq \square 0.05$. Comparison of SCARF1 expression in (B) LUAD and (D) LUSC patients who survived or died tumour-free (blue dots) and those who died with tumour present (red dots). ${ }^{* * *}$ is representative of statistical significance as measured by the Mann Whitney U-test, where $\mathrm{p} \square \leq \square 0.005$. Data in this Figure was generated from the TGCA dataset using the cBioPortal website (https://www.cbioportal.org/) (accessed $28^{\text {th }}$ April 2020). 


\section{Prognostic value of SCARF1 expression in NSCLCs}

Having found that a loss of SCARF1 expression correlates with more advanced and aggressive tumours (Figure 2) and worse outcome (Figure 3) in LUAD, we next sought to investigate its prognostic value in NSCLCs. With regards to overall survival, high expression of SCARF1 was highly indicative of a better prognosis in LUAD $(\mathrm{HR}=0.68,95 \% \mathrm{Cl}=0.52-0.87, p \leq 0.01$; Figure 4A). We also assessed the prognostic value of SCARF1 expression in correlation with a range of clinicopathological features in LUAD. In both male and female patients, higher SCARF1 expression was suggestive of better overall survival (Male $\mathrm{HR}=0.59,95 \%$ $\mathrm{Cl}=0.41-0.85, p \leq 0.005 ;$ Female $\mathrm{HR}=0.61,95 \% \mathrm{Cl}=0.40-0.94, p \leq 0.05$; Figure 4B) $0.61(0.40-0.94)$. With regards to stages of LUAD tumours, higher SCARF1 expression was strongly associated with better overall survival in early stage (Stage I) disease $(\mathrm{HR}=0.27,95 \% \mathrm{Cl}=0.12-0.59, p \leq 0.001$; Figure 4B), but held no prognostic value in more advanced (Stage II) cancers. High expression of SCARF1 was also indicative of improved overall survival in non-smokers $(\mathrm{HR}=0.37$, $95 \% \mathrm{Cl}=0.13-1.03$; Figure 4B), but exhibited no prognostic value in those patients with a history of smoking (Figure 4B). The level of SCARF1 expression held no prognostic value in the presence or absence of lymph node metastasis in LUAD. In keeping with the fact that SCARF1 expression was not associated with tumour biology or outcome in any way, it was unsurprising that SCARF1 showed no prognostic value in those patients with LUSC. 
A

LUAD

Overall Survival

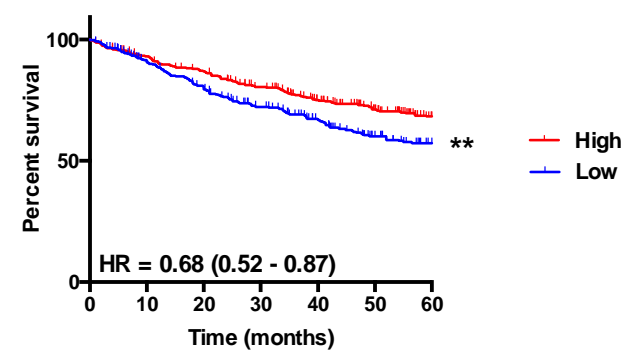

Number at risk

High $\quad \begin{array}{lllllll}358 & 324 & 298 & 264 & 224 & 187 & 147\end{array}$

Low $\quad \begin{array}{lllllll}361 & 312 & 272 & 231 & 198 & 160 & 135\end{array}$

B

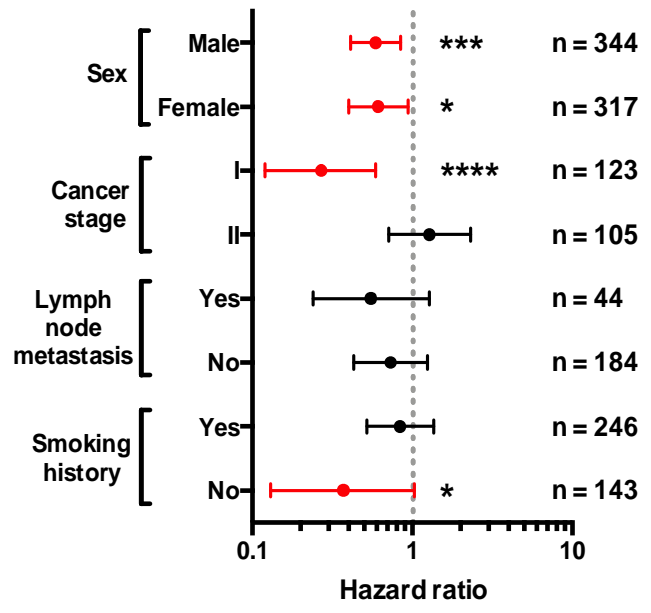

C

LUSC

Overall Survival

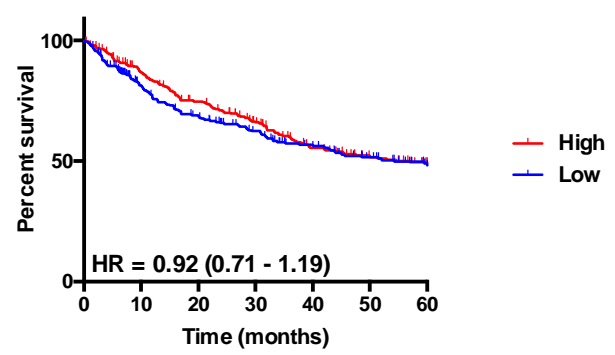

Number at risk

High $\quad \begin{array}{lllllll}262 & 208 & 174 & 144 & 111 & 94 & 80\end{array}$

Low $\quad \begin{array}{lllllll}262 & 183 & 151 & 125 & 103 & 85 & 67\end{array}$

D

LUSC

Overall Survival

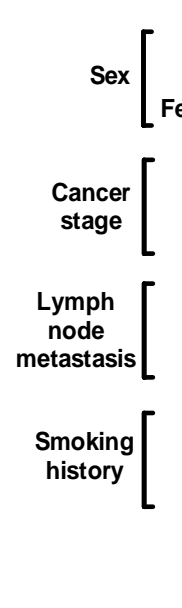

$n=342$

$n=129$

$n=106$

$n=160$

$\mathrm{n}=77$

$n=208$

$n=244$

Figure 4 - SCARF1 expression is predictive of survival in LUAD, but not LUSC. (A) Overall survival in LUAD patients separated into two groups ('High' and 'Low' expression) via the median expression of SCARF1. ** indicates statistical significance where $p \square \leq \square 0.01$. HR = hazard ratio. (B) Forest plots of Overall survival in relation to various clinicopathological features of LUAD patients. Red plots highlight clinicopathological parameters in which statistical significance was achieved. * ${ }^{* * *}$ and ${ }^{* * * *}$ indicate statistical significance where $p \square \leq \square 0.05, p \square \leq \square 0.005$ or $p \square \leq \square 0.001$, respectively. (C) Overall survival in LUSC patients separated into two groups ('High' and 'Low' expression) via the median expression of SCARF1. (D) Forest plots of Overall survival in relation to various clinicopathological features of LUAD patients. Data in this Figure was generated with use of KM Plotter (http://kmplot.com/analysis/). 
SCARF1 is expressed in tumour endothelial cells and correlates with CD4 $4^{+} T$ cell infiltration in LUAD

SCARF1 exhibits a strong endothelial signature in HCC tumours [11] and, given that in homeostatic conditions, SCARF1 is predominantly expressed in normal endothelial cell (NEC) populations within the human lung (Figure 1B), we next explored whether or not it was also expressed in tumour endothelial cells (TEC) of NSCLCs. With use of the publically-available single cell RNA sequencing data from the Lung Endothelial Cell taxonomy (LungECtax) database (https://endotheliomics.shinyapps.io/lung ectax/), we were able to visualise the different endothelial cell populations present in normal human lung and NSCLC tissues. t-Distributed Stochastic Neighbour Embedding (tSNE) plots generated via the LungECtax software showed that there were 13 distinct endothelial cell subtypes present within human lung tissues (Figure $5 \mathrm{~A}$ ) and that certain populations were enriched in either normal or NSCLC tumour tissues (Figure 5B). The LungECTax software was subsequently used to explore the expression pattern of SCARF1 across the various endothelial cell populations. SCARF1 was ubiquitously expressed across throughout all 13 lung endothelial cell populations, showing comparable expression in both NEC- and TEC-enriched populations (Figure 5C). The expression of SCARF1 in NSCLC tumour endothelia was highly consistent with our previous findings of its presence in HCC tumour endothelia.

We have previously shown that SCARF1 expression correlated with the level of $\mathrm{CD}^{+} \mathrm{T}$ cell infiltration in $\mathrm{HCC}$ tumours and hypothesised that it could function to specifically recruit proinflammatory subsets of $\mathrm{CD}^{+} \mathrm{T}$ cells [11]. Given the parallels of SCARF1 in HCC and its expression in LUAD tumours described throughout the current study, we next aimed to investigate whether SCARF1 could play a role in the 
recruitment of tumour-infiltrating lymphocytes (TILs) to the LUAD tumour microenvironment. To explore this, we used a publically-available tool, Estimation of STromal and Immune cells in MAlignant Tumor tissues using Expression data (ESTIMATE; https://bioinformatics.mdanderson.org/estimate/) to correlate SCARF1 expression with immune score, which is representative of immune infiltration in tumour tissues [34]. Using ESTIMATE, we demonstrated a moderate positive correlation between SCARF1 expression and immune score in LUAD tumour tissues (Figure 5D). Next, to explore the relationship of SCARF1 and TILs in more detail, we used Tumor

IMmune Estimation

Resource (TIMER; https://cistrome.shinyapps.io/timer/) to correlate SCARF1 expression with the level of $\mathrm{CD}^{+} \mathrm{T}$ cell infiltration of LUAD tumours. Using TIMER, we first confirmed that SCARF1 expression is absent from tumour cells, as indicated by a negative 'purity' correlation $\left(-0.312, p=1.39 \mathrm{e}^{-12}\right.$; Figure $5 \mathrm{E}$, left panel). Consistent with our previous findings in HCC, we also demonstrated a moderate positive correlation with $\mathrm{CD}^{+} \mathrm{T}$ cell infiltration (purity-corrected partial Spearman's rho value $=0.425, p=1.17 \mathrm{e}^{-22}$; Figure 5E, middle panel) in LUAD. To demonstrate its specificity for CD4 ${ }^{+} \mathrm{T}_{\text {cells }}{ }^{23}$, we also used TIMER to correlate SCARF1 with $\mathrm{CD}^{+} \mathrm{T}$ cell infiltration in LUAD tumours and showed a much weaker correlation (purity-corrected partial Spearman's rho value $=0.107, p=1.85 \mathrm{e}^{-02} ;$ Figure $5 \mathrm{E}$, right panel) 
A

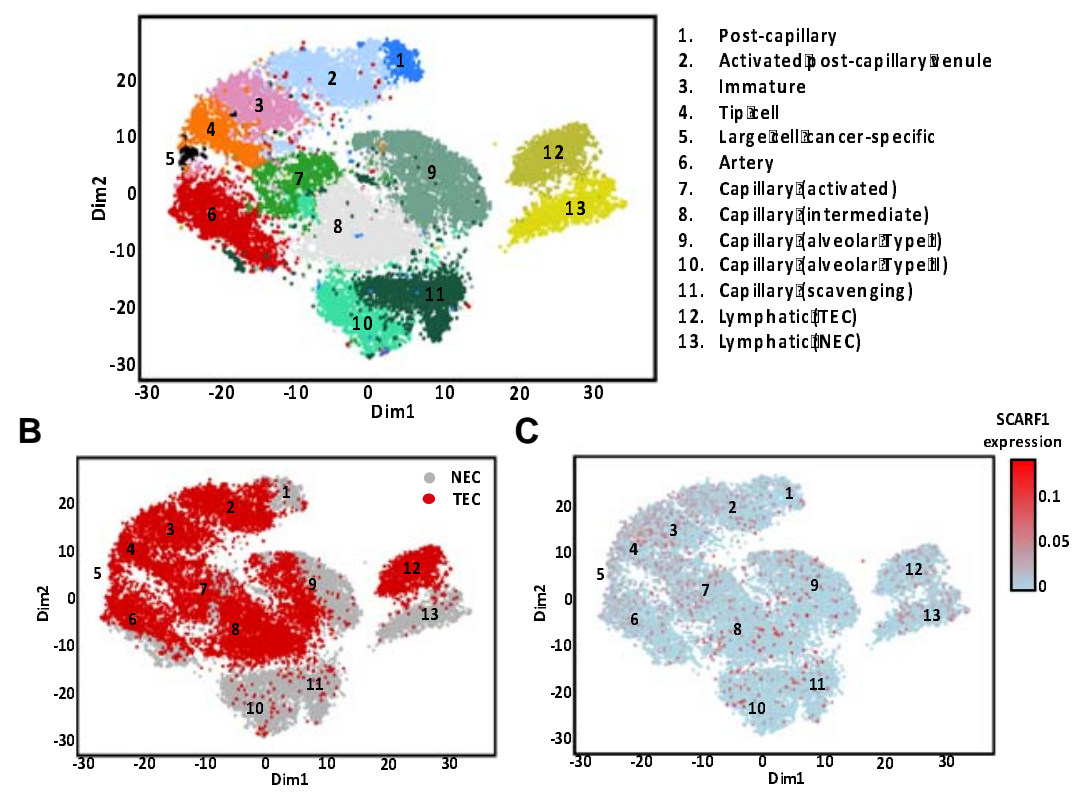

D

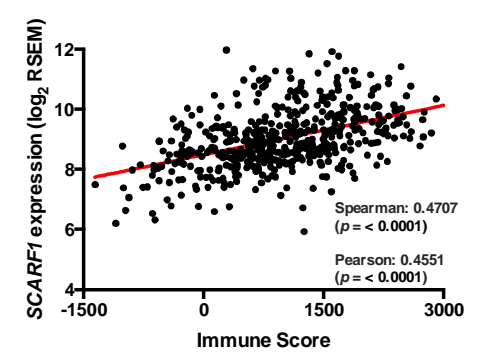

E

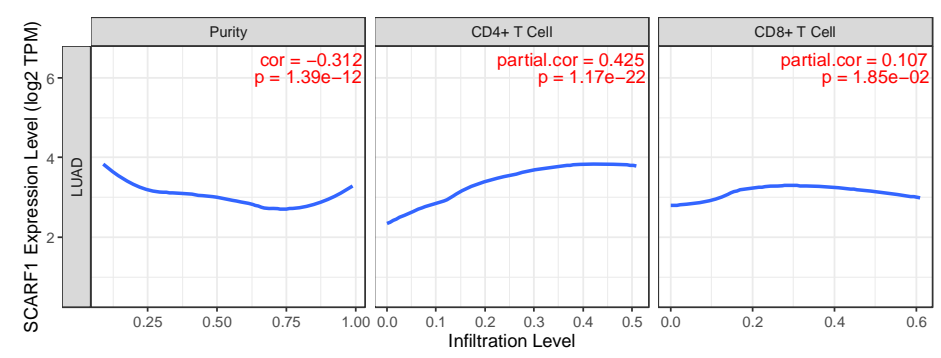

Figure 5 - SCARF1 is widely expressed in NSCLC tumour endothelial cell populations and correlates with $\mathrm{CD4}^{+} \mathrm{T}$ cell infiltration in LUAD. (A) tSNE plot of endothelial cells subtypes present in normal and tumour human lung tissues. (B) tSNE plot of endothelial subtypes enriched in normal endothelial cells (NEC; grey) and tumour endothelial cells (TEC; red). (C) tSNE plot of SCARF1 expression (red) in human lung endothelial cells subtypes. (D) Correlation of SCARF1 expression with immune score in LUAD tumour tissues. (E) Correlation of SCARF1 expression with the extent of $\mathrm{CD}^{+}$and $\mathrm{CD} 8^{+} \mathrm{T}$ cell infiltration in LUAD tumour tissues. tSNE plots in (A), (B) and (C) were generated by the publically-available LungECtax database (https://endotheliomics.shinyapps.io/lung ectax/) accessed on $4^{\text {th }}$ June 2020 . Data in (D) was generated via via Estimation of STromal and Immune cells in MAlignant Tumor tissues using Expression data (ESTIMATE; https://bioinformatics.mdanderson.org/estimate/; accessed 19th August 2020). Data in (E) was generated via the Tumor IMmune Estimation Resource (TIMER; https://cistrome.shinyapps.io/timer/; accessed 12th May 2020). 


\section{Discussion}

Genetic and molecular profiling of tumour tissues has aided the development of highly targeted and more effective treatments for lung cancers, significantly increasing patient survival time; however, lung cancer remains to be a major cause of mortality worldwide. Therefore, further investigation of the tumour microenvironment is warranted to identify novel biomarkers and therapies. Scavenger receptors have previously been show to bind a range of oncogenic ligands, such as heat shock proteins (HSPs) $[35,36]$ and bacterial lipopolysaccharide (LPS) [37-39] and are known to be involved in the pathophysiology of a range of cancers [40]. Indeed, we have previously explored two such receptors, SCARF1 [11] and stabilin-1 [41], in the context of hepatocellular carcinoma (HCC) and hypothesised that they could play important roles in shaping the tumour microenvironment. Scavenger receptors in the lung are known to mediate key innate immune responses to a number of microbial infections $[13,14]$ and environmental antigens [15-17]; however, the study of these receptors in the context of lung cancers remains relatively unexplored.

Recent work from our lab has focussed on the expression and function of a largely understudied scavenger receptor [23], SCARF1 (scavenger receptor class F, member 1), in human liver tissues [11,22]; however, others have also identified its presence in human lung tissues [18]. Here, we show that the lungs are a site of significant SCARF1 expression (Figure 1A) and, like the liver [22], endothelial cells account for the majority of its expression (Figure 1B). In addition, consistent with our findings in gastrointestinal cancers, such as HCC and colonic adenocarcinoma [11], we showed here that SCARF1 expression is significantly downregulated in two major histological subtypes of non-small cell lung cancer (NSCLC), lung adenocarcinoma 
(LUAD) and lung squamous cell carcionoma (LUSC) (Figures 1C-E). In addition, and once again consistent with our previous findings in HCC, we showed that higher intratumoural expression of SCARF1 in LUAD tumour tissues was associated with less aggressive tumour biology (Figure 2) and better outcomes (Figure 3); however, in stark contrast, this association was not evident in LUSC tumours (Figures 2 \& 3). Furthermore, from a prognostic perspective, higher SCARF1 expression in LUAD tumours was highly indicative of better overall survival (Figure 4), but showed no prognostic value in LUSC tumours.

Endothelial-expressed scavenger receptors are known to possess a secondary function as atypical adhesion molecules in the leukocyte recruitment cascade [42] and we have recently described that SCARF1 is potentially involved in the recruitment of proinflammatory $\mathrm{CD}^{+} \mathrm{T}$ cells to the microenvironment of $\mathrm{HCC}$ tumours [11]. Thus, the disparities in the data between the two subtypes of NSCLC described in this study may be explained by the immune status of the two tumours types. LUAD tumours are known to possess a high clonal neoantigen burden [43], which is associated with an inflamed tumour microenvironment enriched in activated effector T cells [43] and higher expression of genes involved in antigen presentation, T cell migration and effector $T$ cell function $[43,44]$. In stark contrast, LUSC tumours exhibit a low neoantigen burden [43] and are known to actively repress key genes in immunity, such as MHC molecules and chemokines, in early stage disease [44]. Ultimately, LUAD tumours may promote a more proinflammatory tumour microenvironment, whereas LUSC tumours are more likely to present as antiinflammatory to escape immune surveillance; therefore, we hypothesised that SCARF1 could function in LUAD tumours as it does in HCC. To explore this, we first needed to show that SCARF1 was indeed present in tumour endothelial cells and we 
were able to do so via the publically-available Lung Endothelial Cell taxonomy (LungECtax) database (Figure $5 \mathrm{~A}-\mathrm{C})$. Next, we showed a positive correlation between SCARF1 expression and immune infiltration score (Figure 5D), and more specifically, the level of $\mathrm{CD}^{+} \mathrm{T}$ cell infiltration in LUAD tumours (Figure 5E, middle panel). Finally, we corroborated our previous findings of SCARF1's specificity for $\mathrm{CD}^{+} \mathrm{T}$ cells [22], by demonstrating a negligible correlation between LUADexpressed SCARF1 and $\mathrm{CD}^{+} \mathrm{T}$ cell infiltration (Figure $5 \mathrm{E}$, right panel). This is particularly relevant to the context of LUAD, as previous studies have shown that an increased prevalence of tumour-infiltrating lymphocytes is a prognostic factor in NSCLCs $[45,46]$. Therefore, increasing the inflammatory status via a SCARF1mediated pathway could have a significant impact on patient outcome.

Although this study represents the first detailed investigation of SCARF1 in human lung tissues and their associated malignancies, this work is still very preliminary as it has only been undertaken with publically-available RNA sequencing data. Therefore, future work should initially focus on corroborating these data with protein expression experiments on ex vivo sections of human lung tissue. In addition, flow-based adhesion assays with primary lung endothelial cells, as we have previously performed with liver endothelial cells [47], would confirm functionality of lung-expressed SCARF1. Should this be consistent with the data presented in the current study, then further in vivo work would then be required with SCARF1 knockout models to fully confirm its contribution to the NSCLC immune microenvironment. Taken all together, our data presented here describes a potential beneficial role for SCARF1 in LUAD tumours. The current study, along with previous data, further emphasises a role for SCARF1 in shaping the tumour microenvironment 
and is highly suggestive that SCARF1 present novel therapeutic target in the treatment of immunologically active cancers.

\section{Conflicts of Interest}

SS has received a research grant from Faron Pharmaceuticals to design a Phase I/II trial (TIETALC) of the drug "Clevergen" in patients with HCC. SS also reports consulting for Faron Pharmaceuticals. The remaining authors declare that the research was conducted in the absence of any commercial or financial relationships that could be construed as a potential conflict of interest. The funders had no role in the design of the study; in the collection, analyses, or interpretation of data; in the writing of the manuscript, or in the decision to publish the results.

\section{Author Contributions}

Conceptualization, DAP; Data curation, DAP; Formal analysis, DAP; Funding acquisition, DAP and SS; Investigation, DAP; Methodology, DAP; Writing - original draft, DAP; Writing - review \& editing, DAP and SS.

\section{Funding}

DAP and SS are funded by a Medical Research Council Project Grant (MR/R010013/1).

\section{Acknowledgments}

This paper presents independent research supported by the Birmingham NIHR Liver Biomedical Research Unit based at the University Hospitals Birmingham NHS Foundation Trust and the University of Birmingham. The views expressed are those of the authors and not necessarily those of the NHS, the NIHR or the Department of Health. 


\section{References}

1. Bray, F., Ferlay, J., Soerjomataram, I., Siegel, R.L., Torre, L.A. and Jemal A. Global cancer statistics 2018: GLOBOCAN estimates of incidence and mortality worldwide for 36 cancers in 185 countries. CA Cancer J Clin 2018, 68, 394-424

2. Hirsch, F.R., Scagliotti, G.V., Mulshine, J.L., Kwon, R., Curran Jr, W.J., Wu, Y.L. et al. Lung cancer: current therapies and new targeted treatments. The Lancet 2017, 389, 299-311

3. Herbst, R.S., Morgensztern, D. and Boshoff, C. The biology and management of non-small cell lung cancer. Nature 2018, 553, 446-454

4. Denisenko, T.V., Budkevich, I.N. and Zhivotovsky, B. Cell death-based treatment of lung adenocarcinoma. Cell Death Dis 2018, 9, 1-14

5. Cancer Genome Atlas Research Network, Comprehensive genomic characterization of squamous cell lung cancers. Nature 2012, 489, 519-525

6. Cancer Genome Atlas Research Network, 2014. Comprehensive molecular profiling of lung adenocarcinoma. Nature 2014, 511, 543-550

7. Dai, F., Liu, L., Che, G., Yu, N., Pu, Q., Zhang, S. et al. The number and microlocalization of tumor-associated immune cells are associated with patient's survival time in non-small cell lung cancer. BMC Cancer 2010, 10, 220.

8. Bremnes, R.M., Busund, L.T., Kilvær, T.L., Andersen, S., Richardsen, E., Paulsen, E.E., et al. The role of tumor-infiltrating lymphocytes in development, progression, and prognosis of non-small cell lung cancer. J Thor Oncol 2016, $11,789-800$

9. Navab, R., Strumpf, D., Bandarchi, B., Zhu, C.Q., Pintilie, M., Ramnarine, V.R. et al. Prognostic gene-expression signature of carcinoma-associated fibroblasts in non-small cell lung cancer. Proc Natl Acad Sci, 2011, 108, 71607165

10.Chen, W.J., Ho, C.C., Chang, Y.L., Chen, H.Y., Lin, C.A., Ling, T.Y. et al. Cancer-associated fibroblasts regulate the plasticity of lung cancer stemness via paracrine signalling. Nat Commun, 2014, 5,1-17

11. Patten, D.A., Wilkinson, A., O'Rourke, J. and Shetty, S. Prognostic value and potential immunoregulatory role of SCARF1 in hepatocellular carcinoma. Front Oncol 2020, 10, 565950

12. Canton J, Neculai D, Grinstein S. Scavenger receptors in homeostasis and immunity. Nat Rev Immunol 2013, 13, 621-634

13. Arredouani, M.S., Yang, Z., Imrich, A., Ning, Y., Qin, G. and Kobzik, L. The macrophage scavenger receptor SR-Al/II and lung defense against pneumococci and particles. Am J Resp Cell Mol 2006, 35, 474-478

14. Blanchet, C., Jouvion, G., Fitting, C., Cavaillon, J.M. and Adib-Conquy, M. Protective or deleterious role of scavenger receptors SR-A and CD36 on host resistance to Staphylococcus aureus depends on the site of infection. PloS One 2014, 9

15. Thakur, S.A., Hamilton Jr, R.F. and Holian, A. Role of scavenger receptor a family in lung inflammation from exposure to environmental particles. J Immunotoxicol 2008, 5, 151-157 
16. Arredouani, M.S., Franco, F., Imrich, A., Fedulov, A., Lu, X., Perkins, D. et al Scavenger Receptors SR-AI/II and MARCO limit pulmonary dendritic cell migration and allergic airway inflammation. J Immunol 2007, 178, 5912-5920.

17. Dahl, M., Bauer, A.K., Arredouani, M., Soininen, R., Tryggvason, K., Kleeberger, S.R. et al. Protection against inhaled oxidants through scavenging of oxidized lipids by macrophage receptors MARCO and SR-AI/II. $\mathrm{J}$ Clin Invest 2007, 117, 757-764

18. Ishii, J., Adachi, H., Aoki, J., Koizumi, H., Tomita, S., et al. SREC-II, a new member of the scavenger receptor type $F$ family, trans-interacts with SREC-I through its extracellular domain. J Biol Chem. 2002, 277, 39696-702.

19. Means, T.K., Mylonakis, E., Tampakakis, E., Colvin, R.A., Seung, E., Puckett, L., et al. Evolutionarily conserved recognition and innate immunity to fungal pathogens by the scavenger receptors SCARF1 and CD36. J Exp Med 2009, 206, 637-653 (2009)

20. Ramirez-Ortiz, Z.G., Pendergraft III, W.F., Prasad, A., Byrne, M.H., Iram, T., Blanchette, C.J., et al. The scavenger receptor SCARF1 mediates the clearance of apoptotic cells and prevents autoimmunity. Nat Immunol 2013, $14,917-926$

21. Adachi, H., Tsujimoto, M., Arai, H., Inoue, K. Expression cloning of a novel scavenger receptor from human endothelial cells. J Biol Chem 1997, 272, 31217-31220

22. Patten, D.A., Kamarajah, S.K., Rose, J.M., Tickle, J., Shepherd, E.L., Adams, D.H., et al. SCARF-1 promotes adhesion of CD4 ${ }^{+} \mathrm{T}$ cells to human hepatic sinusoidal endothelium under conditions of shear stress. Sci Rep 2017, 7, 17600

23. Patten, D.A. SCARF1: a multifaceted, yet largely understudied, scavenger receptor. Inflam Res 2018, 67, 627-632

24. Wicker-Planquart, C., Dufour, S., Tacnet-Delorme, P., Bally, I., Delneste, Y., Frachet, P., et al. Molecular and Cellular Interactions of Scavenger Receptor SR-F1 With Complement C1q Provide Insights Into Its Role in the Clearance of Apoptotic Cells. Front Immunol 2020, 11, 544

25. Beauvillain, C., Meloni, F., Sirard, J.-C., Blanchard, S., Jarry, U., Scotet, M., et al. The scavenger receptors SRA-1 and SREC-I cooperate with TLR2 in the recognition of the hepatitis $C$ virus non-structural protein 3 by dendritic cells. $J$ Hepatol 2010, 52, 644-651

26. Murshid, A., Gong, J., Ahmad, R., Borges, T.J., Calderwood, S.K. Scavenger receptor SREC-I promotes double stranded RNA-mediated TLR3 activation in human monocytes. Immunobiol 2015, 220, 823-832

27. Piccolo, P., Vetrini, F., Mithbaokar, P., Grove, N.C., Bertin, T., Palmer, D., et al. SR-A and SREC-I are Kupffer and endothelial cell receptors for helperdependent adenoviral vectors. Mol Ther 2013, 21, 767-74

28. Rechner, C., Kühlewein, C., Müller, A., Schild, H., Rudel, T. Host glycoprotein Gp96 and scavenger receptor SREC interact with PorB of disseminating Neisseria gonorrhoeae in an epithelial invasion pathway. Cell Host Microbe 2007, 2, 393-403

29. Jeannin, P., Bottazzi, B., Sironi, M., Doni, A., Rusnati, M., Presta, M., et al. Complexity and complementarity of outer membrane protein $A$ recognition by cellular and humoral innate immunity receptors. Immunity 2005, 22, 551-560

30. Baur, S., Rautenberg, M., Faulstich, M., Grau, T., Severin, Y., Unger, C. et al. A nasal epithelial receptor for Staphylococcus aureus WTA governs adhesion 
to epithelial cells and modulates nasal colonization. PLoS Pathog 2014, 10, e1004089

31. Murshid, A., Gong, J., Prince, T., Borges, T.J., Calderwood, S.K. Scavenger receptor SREC-I mediated entry of TLR4 into lipid microdomains and triggered inflammatory cytokine release in RAW 264.7 cells upon LPS activation. PloS One 2015, 10, e0122529

32. Taylor, A.M., Shih, J., Ha, G., Gao, G.F., Zhang, X., Berger, A.C., et al. Genomic and functional approaches to understanding cancer aneuploidy. Cancer Cell 2018, 33, 676-689

33. Buffa, F., Harris, A., West, C., Miller, C. Large meta-analysis of multiple cancers reveals a common, compact and highly prognostic hypoxia metagene. Br J Cancer 2010, 102, 428-435

34. Yoshihara, K., Shahmoradgoli, M., Martínez, E., Vegesna, R., Kim, H., TorresGarcia, W., et al. Inferring tumour purity and stromal and immune cell admixture from expression data. Nat Commun 2013, 4, 1-11

35. Calderwood, S.K., Gong, J. Heat shock proteins promote cancer: it's a protection racket. Trends Biochem Sci 2016, 41, 311-323

36. Wu, J., Liu, T., Rios, Z., Mei, Q., Lin, X., Cao, S. Heat shock proteins and cancer. Trends Pharmacol Sci 2017, 38, 226-256

37. Ikebe, M., Kitaura, Y., Nakamura, M., Tanaka, H., Yamasaki, A., Nagai, S., et al. Lipopolysaccharide (LPS) increases the invasive ability of pancreatic cancer cells through the TLR4/MyD88 signaling pathway. J Surg Oncol 2009, $100,725-731$

38. Hsu, R.Y., Chan, C.H., Spicer, J.D., Rousseau, M.C., Giannias, B., Rousseau, S., et al. LPS-induced TLR4 signaling in human colorectal cancer cells increases $\beta 1$ integrin-mediated cell adhesion and liver metastasis. Cancer Res 2011, 71, 1989-1998

39. Kisseleva, T., Song, L., Vorontchikhina, M., Feirt, N., Kitajewski, J., Schindler, C. NF-kB regulation of endothelial cell function during LPS-induced toxemia and cancer. J Clin Invest 2006, 116, 2955-2963

40.Yu, X., Guo, C., Fisher, P.B., Subjeck, J.R., Wang, X.-Y. Scavenger receptors: emerging roles in cancer biology and immunology. Adv Cancer Res 2015, 128, 309-364

41. Shetty, S., Weston, C.J., Oo, Y.H., Westerlund, N., Stamataki, Z., Youster, J., et al. Common lymphatic endothelial and vascular endothelial receptor-1 mediates the transmigration of regulatory $\mathrm{T}$ cells across human hepatic sinusoidal endothelium. J Immunol 2011, 186, 4147-4155

42. Patten, D.A. and Shetty, S. More than just a removal service: scavenger receptors in leukocyte trafficking. Front Immunol 2018, 9, 2904

43. McGranahan, N., Furness, A.J.S., Rosenthal, R., Ramskov, S., Lyngaa, R., Saini, S.K. et al. Clonal neoantigens elicit $T$ cell immunoreactivity and sensitivity to immune checkpoint blockade. Science 2016, 351, 1463-1469

44. Chen, M., Liu, X., Du, J., Wang, X.J. and Xia, L., 2017. Differentiated regulation of immune-response related genes between LUAD and LUSC subtypes of lung cancers. Oncotarget 2017, 8, 133.

45. Brambilla E, Le Teuff G, Marguet S, et al. Prognostic effect of tumor lymphocytic infiltration in resectable non-small-cell lung cancer. J Clin Oncol. 2016, 34, 1223-1230 
46.Zeng DQ, Yu YF, Ou QY, et al. Prognostic and predictive value of tumor-infiltrating lymphocytes for clinical therapeutic research in patients with non-small cell lung cancer. Oncotarget 2016, 7, 13765-13781

47. Shetty, S., Weston, C.J., Adams, D.H., Lalor, P.F. A flow adhesion assay to study leucocyte recruitment to human hepatic sinusoidal endothelium under conditions of shear stress. JoVE 2014, 85 